\title{
AVALIAÇÃO DO CICLO DE VIDA (ACV) APLICADA A REMEDIAÇÃO DE ÁREAS CONTAMINADAS
}

\section{Claudia Echevenguá Teixeira}

Doutora em Engenharia Civil e Ambiental pela Universidade de Sherbooke, Canada Pesquisadora do Instituto de Pesquisas Tecnológicas do Estado de São Paulo - IPT/SP

Professora da Universidade Nove de Julho - UNINOVE ceteixeira10@gmail.com

Ana Carolina La Laina Cunha

Mestre em Tecnologia Ambiental pelo Instituto de Pesquisas Tecnológicas - IPT/SP carollalaina@ig.com.br

\author{
Rachel Horta Arduin \\ Mestranda em Engenharia Ambiental da Universidade de São Paulo - USP \\ Pesquisadora do Instituto de Pesquisas Tecnológicas do Estado de São Paulo - IPT/SP \\ rachel@ipt.br \\ Mauro Silva Ruiz \\ Doutor em Geografia pela Southern Illinois University at Carbondale, Estados Unidos \\ Professor da Universidade Nove de Julho - UNINOVE \\ maurosilvaruiz@uninove.br
}

\section{RESUMO}

A remediação de áreas contaminadas é a aplicação de técnica(s) visando à remoção, redução ou contenção de contaminantes. Os processos de remediação podem acarretar impactos ambientais associados ao uso de recursos, emissões e transformação da área. Este artigo apresenta o levantamento do estado da arte referente ao uso da ACV aplicada a tecnologias de remediação, bem como o contexto de remediação de áreas contaminadas no Brasil. Esta pesquisa foi conduzida com base em uma revisão bibliográfica sistemática. Os critérios envolveram $o$ estabelecimento de palavras-chave para a busca de informações, a definição dos meios e plataformas de busca (bases de dados), bem como classificação e sistematização. O levantamento de dados procedeu-se até o mês de dezembro de 2011. Trinta e sete estudos foram localizados, entre 1997 e 2011. Destes, trinta artigos indexados em periódicos científicos internacionais e sete de outros fontes. A unidade funcional e o escopo temporal variaram, dependendo do cenário da contaminação e dos critérios técnicos estabelecidos para a remediação. Para a avaliação do impacto do ciclo de vida, a maior parte dos estudos utilizou metodologias de impacto de ponto médio, sendo que a categoria de impacto de maior incidência foi o potencial de aquecimento global. A aplicação da ACV nos estudos de casos pesquisados foi considerada um método que permitiu mapear os impactos ambientais associados às tecnologias de remediação, apesar de limitações quanto aos inventários e a complexidade dos processos.

Palavras-chave: Áreas contaminadas; Avaliação do ciclo de vida [ACV]; Impacto ambiental; Remediação.

\section{LIFE CYCLE ASSESSMENT (LCA) APPLIED TO REMEDIATION TECHNOLOGIES}

\begin{abstract}
The remediation of contaminated areas requires the application of techniques to remove, reduce, or contain contaminants. The processes of remediation can result in environmental impacts associated with resource use, emissions, and transformation of the area. This article presents a survey of the technique of using LCA to map remediation technologies, as well as the context of remediation of contaminated areas in Brazil. The research was carried out based on a systematic literature review. The criteria involved the establishment of keywords to search for information, definition of media, and search platforms (databases), as well as classification and systematization. Data were collected until December 2011. Thirty-seven published studies were located, mainly in journals, between 1997 and 2011. The functional units of time and scope varied depending on the scenario of contamination and the technical criteria established for the remediation. To evaluate the impact of the life cycle, most of the studies used midpoint impact methodologies. The global warming potential was the impact category with the highest incidence of midpoints. The application of LCA in the case studies surveyed was considered a method that allowed mapping of the environmental impacts associated with remediation technologies, even considering the existing limitations of the complexity of processes and inventories.
\end{abstract}

Key words: Life cycle assessment [LCA]; Contaminated sites; Remediation; Environmental impact.

* Apoio recebido do Fundo de Apoio a Pesquisa - FAP/UNINOVE. 


\section{INTRODUÇÃO}

A destinação inadequada de resíduos e de substâncias químicas no solo e nos recursos hídricos acarreta impactos negativos sobre a saúde humana e ao ambiente. Inúmeros casos de contaminação por substâncias químicas e resíduos perigosos vêm sendo relatados no Brasil e no mundo (e.g. Côrtes, Alves Filho, Ruiz e Teixeira, 2011; Oliveira, Bastos, Dias, Silva e Medeiros, 2003; Phillips, Hung e Bosela, 2007). Entre os grupos de substâncias químicas responsáveis por ações adversas à saúde e ao meio ambiente, quando disseminadas indiscriminadamente, encontramse os organoclorados, as bifenilas policloradas, os compostos fenólicos, os hidrocarbonetos de petróleo, os metais pesados, entre outros.

No estado de São Paulo, 4.131 áreas estão catalogadas pela Companhia Ambiental do Estado de São Paulo [Cetesb] como áreas sob investigação, contaminadas, em processo de reabilitação ou reabilitadas. Deste total, 1.245 (30,1\%) estão sob investigação, $1.835(44,4 \%)$ estão contaminadas, $787(19,1 \%)$ em processo de reabilitação e $264(6,4 \%)$ consideradas reabilitadas (Cetesb, 2011).

Restaurar a multifuncionalidade de uma área contaminada pode levar décadas e ser inviável em termos de valor do investimento. A abordagem atual em projetos de descontaminação é pautada no gerenciamento de riscos à saúde humana e ao meio ambiente (Beinat e Nijkamp,1997).

A remediação de uma área contaminada pode envolver processos biológicos, térmicos, físicos e químicos, como, por exemplo, biorremediação, fitorremediação, tratamento químico e térmico, entre outros (Sharma e Reddy, 2004). A adoção de uma ou a associação de diferentes tecnologias vai depender do uso pretendido para a área contaminada, da avaliação de risco, eficiência do processo, tempo para descontaminação e viabilidade econômica. Apesar de o processo de reabilitação de uma área contaminada sanar questões ambientais e de saúde pública, também pode gerar cargas ambientais negativas. As técnicas de remediação podem durar um longo período de tempo e são responsáveis pelo consumo de recursos naturais, emissão de gases e efluentes, gerações de resíduos, uso da terra, entre outros. Os efeitos de um processo de descontaminação no meio ambiente e na saúde humana são também importantes aspectos a serem considerados quando da definição das tecnologias de remediação (Beinat e Nijkamp,1997).

Com o objetivo de fomentar práticas sustentáveis em remediação, foi realizado, em 2006, nos Estados Unidos, o The Sustainable Remediation Forum [SURF], iniciando um diálogo sobre remediação sustentável entre os atores envolvidos na decisão e implantação de técnicas de remediação. $\mathrm{O}$ fórum suscitou a formação de grupos de remediação sustentável além das fronteiras desse país.

A United States Environmental Protection Agency [US EPA] define "green remediation" ou "remediação verde" como a prática que considera todos os efeitos ambientais de uma remediação em cada fase do processo, e incorpora estratégias para maximizar o benefício ambiental da remediação. Ou seja, além de reduzir o nível de contaminação a um nível aceitável, reduz também a carga ambiental associada ao consumo de recursos e emissões de poluentes das tecnologias de remediação em si. Entre os métodos que vêm sendo utilizados no anseio de avaliar e aplicar tecnologias de remediação menos agressivas ao ambiente, destacam-se a Avaliação do Ciclo de Vida [ACV], Análise Multicritério [AMC], Análise de Custo-Benefício [ACB] e a Análise de Custo-Efetividade [ACE]. O primeiro estudo de ACV aplicado a tecnologias de remediação foi publicado, em formato de relatório técnico, por Beinat e Nijikamp em 1997. Nesse relatório estão relatados os aspectos de redução de risco, mérito ambiental e custos para avaliação de tecnologias de remediação. Desde então, muitos pesquisadores, pelo princípio de processos ambientalmente corretos, têm utilizado a ACV para avaliar e estimar os impactos ambientais associados às tecnologias de remediação (e.g. Higgins e Olson, 2009; Inoue e Katayama, 2011; Suer \& Andersson-Sköld, 2011; Volkwein, Hurting e Klöpffer, 1999). No Brasil, as iniciativas para avaliar as cargas ambientais associadas às tecnologias de remediação ainda são pouco expressivas. $\mathrm{O}$ tema de sustentabilidade em remediação de áreas contaminadas foi introduzido no Brasil por intermédio 
da Rede Latino Americana de Prevenção e Gestão de Sítios Contaminados [ReLASC].

Neste cenário, este trabalho visou sistematizar e avaliar o uso da ACV no contexto de remediação de áreas contaminadas. O levantamento do estado da arte foi conduzido com base no princípio do método de revisão bibliográfica sistemática (Brereton, Kitchenham, Budgen, Turner e Khalil, 2007; Pigosso, 2008). A questão central de pesquisa foi: A ACV é utilizada para avaliar tecnologias de remediação? Além desta, questionou-se: Se sim, para quais situações de remediação? Qual a estrutura da ACV em tecnologias de remediação? Como estabelecer o escopo? Quais os parâmetros de inventário? Como avaliar os impactos? Neste contexto, a partir de uma revisão bibliográfica sistemática, este artigo apresenta o cenário internacional da aplicação da avaliação do ciclo de vida em tecnologias de remediação.

Este trabalho está estruturado da seguinte forma: na seção dois, a revisão da literatura; na seção três, o método da pesquisa para o levantamento e sistematização de dados, na seção quatro, os resultados e discussões, e na seção cinco, a conclusão e as recomendações para futuras pesquisas.

\section{FUNDAMENTAÇÃO TEÓRICA}

Essa seção apresenta o estado da arte e os principais conceitos das áreas do conhecimento relacionadas a essa pesquisa, a saber: gerenciamento de áreas contaminadas no Brasil, remediação de áreas contaminadas, sustentabilidade em remediação e avaliação do ciclo de vida.

\subsection{Gerenciamento de áreas contaminadas no Brasil}

De acordo com a Lei Estadual $n^{\circ} 13.577$, de 8 de julho de 2009, área contaminada é uma área, terreno, local, instalação, edificação ou benfeitoria que contenha quantidades ou concentrações de matéria em condições que causem ou possam causar danos à saúde humana, ao meio ambiente ou a outro bem a proteger (São Paulo, 2009). No artigo sexto da resolução do Conselho Nacional de Meio Ambiente [Conama] $n^{\circ}$ 420, a contaminação é descrita como a presença de substância(s) química(s) no ar, água ou solo, decorrentes de atividades antrópicas, em concentrações tais que restrinjam a utilização desse recurso ambiental para os usos atuais ou pretendidos, definidos com base em avaliação de risco à saúde humana, assim como aos bens a proteger, em cenário de exposição padronizado ou específico (Conama, 2009).

Uma área contaminada seja por produção industrial, produção agrícola, mineração, armazenamento ou uso de elementos contaminantes, representa um passivo ambiental. $\mathrm{O}$ termo "passivo ambiental" pode ser definido como uma obrigação adquirida em decorrência de transações anteriores ou presentes, que provocaram ou provocam danos ao meio ambiente ou a terceiros, de forma voluntária ou involuntária, que deverão ser indenizados por meio de entrega de benefícios econômicos ou prestação de serviços em um momento futuro (Galdino, Santos, Pinheiro, Marques Junior \& Ramos, 2004).

No Brasil, o Estado de São Paulo, por meio da Companhia Ambiental do Estado de São Paulo (Cetesb), foi pioneiro no estabelecimento de um procedimento de gerenciamento de áreas contaminadas desde 2000, sendo o procedimento aprimorado e consolidado pela Decisão de Diretoria No 103/2007/C/E, (Cetesb, 2007). A Resolução Conama $n^{\circ} 420$ foi um marco regulatório importante na busca de solucionar o problema de áreas contaminadas no território nacional, uma vez que esta estabelece os critérios e valores orientadores de qualidade do solo quanto à presença de substâncias químicas e diretrizes para o gerenciamento ambiental de áreas contaminadas. Segundo a referida resolução, o gerenciamento de áreas contaminadas deve conter procedimentos e ações voltadas a eliminar o perigo ou reduzir o risco à saúde humana; eliminar ou minimizar os riscos ao meio ambiente; evitar danos aos demais bens a proteger; evitar danos ao bem-estar público durante a execução de ações para reabilitação; e possibilitar o uso declarado ou futuro da área, observando o planejamento de uso e ocupação do solo (Conama, 2009).

Em termos técnicos, o gerenciamento de áreas contaminadas é uma sequência de etapas que têm como objetivo identificar e estabelecer a extensão da contaminação e desenvolver ações de 
recuperação, após a confirmação da contaminação. $O$ processo de recuperação envolve a investigação detalhada, avaliação de risco, investigação de remediação, projeto e execução da remediação e monitoramento (Cetesb, 2007).

Na decisão de diretoria No 103/2007/C/E, de 22 de junho de 2007 (Cetesb, 2007), o risco referente a uma área contaminada [AC] é definido como a probabilidade de um efeito adverso aos bens a proteger em decorrência de uma exposição aos contaminantes presentes em uma AC. No caso de risco à saúde ou risco ecológico, medidas de controle institucional e de engenharia são implantadas e a área deve ser submetida à remediação por meio de tecnologias disponíveis. Situações de perigo requerem ação emergencial como, por exemplo, eliminar qualquer tipo de contato da população com a área.

\subsection{Remediação de áreas contaminadas}

A remediação é definida pela resolução Conama n 420 , como uma das ações de intervenção para reabilitação de área contaminada, que consiste em aplicação de técnicas, visando à remoção, contenção ou redução das concentrações de contaminantes (Conama, 2009).

A remediação não necessariamente recupera a área contaminada em sua totalidade. Segundo Beinat e Van Drunen (1997), restaurar a multifuncionalidade de uma área tem demonstrado alguns pontos negativos. Primeiro, os custos dessa recuperação podem ser extremamente altos, além disso, algumas alternativas de remediação atingem resultados muito bons em termos de qualidade do solo, mas elas simplesmente transferem a contaminação para outro meio. Tecnicamente, a remediação de áreas contaminadas depende do tipo de contaminante, de como o contaminante está disposto no ambiente e das características do contaminante, ambiente e entorno.

Atualmente, há uma gama de tecnologias de remediação de distintos mecanismos, que podem envolver processos biológicos, térmicos, físicos e químicos, como biorremediação, fitorremediação, oxidação química, dessorção térmica, entre outros (Sharma e Reddy, 2004). O uso ou associação de diferentes tecnologias depende da avaliação de risco, que define as metas de remediação e a eficiência necessária de descontaminação, tempo e viabilidade econômica.

As tecnologias de remediação de áreas contaminadas podem ser para aplicação in situ, em que a remediação atua na zona onde o contaminante se encontra; ex situ, que consiste em retirar o meio contaminado (solo e/ou água) e tratá-lo em algum local específico; e ainda ex situ e on site, em que o material contaminado é retirado e o tratamento ocorre na própria área (Sharma e Reddy, 2004).

A aplicação da tecnologia de remediação pode tanto ser direcionada à fonte ou à pluma de contaminação, ou à combinação dos dois. Enquanto para remediação de fontes de contaminantes geralmente são utilizadas tecnologias mais agressivas e de curto prazo, as tecnologias para remediação da pluma são geralmente de longo prazo, almejando o controle ou tratamento dos contaminantes remanescentes (Lemming, Hauschild, Bjerg, 2010).

\subsection{Sustentabilidade em remediação}

A abordagem na tomada de decisão em áreas contaminadas mudou substancialmente nos últimos 40 anos. De uma abordagem centrada em custo em meados da década de 1970, tornou-se uma abordagem de estudos de viabilidade tecnológica em meados da década de 1980, seguida de uma abordagem baseada em risco em meados da década de 1990. Por sua vez, a partir de 2000 observa-se que as decisões ambientais devem ser socialmente robustas, em um contexto de desenvolvimento sustentável (Pollard et al., 2004).

Remediação sustentável é amplamente definida pelo SURF como a técnica ou a combinação das técnicas de remediação que pondera a melhor combinação considerando fatores ambientais, sociais e econômicos (Bayer e Finkel, 2006).

Onwubuya et al. (2009) realizaram uma revisão crítica dos métodos de suporte à tomada de 
decisão, visando a aplicação de tecnologias de remediação menos agressivas, sendo apresentados: ACV, AMC, ACB e ACE. Segundo Pollard, Davies, Coley e Lemon (2008), a ACV e a AMC são métodos que têm sido amplamente utilizadas para a coleta de informações detalhadas em aspectos de suporte a decisões ambientais em ecologia industrial e análise de sistemas ambientais. Onwubuya et al. (2009) destacam que as diretrizes do projeto, bem como a avaliação de risco e a viabilidade técnica e a sustentabilidade são fatores chave na seleção de técnicas de remediação na União Europeia.

\subsection{Avaliação do Ciclo de Vida (ACV)}

A Avaliação de Ciclo de Vida (ACV) é um método que permite compilar os fluxos de massa e de energia, de entrada e saída, e avaliar os potenciais impactos ambientais associados a um produto/processo ao longo de todo o seu ciclo de vida (Associação Brasileira de Normas Técnicas [ABNT], 2009a). Na definição da NBR ISO 14040, produto é qualquer bem ou serviço (ABNT, 2009a).

No Brasil, este método está atualmente descrito pelas normas NBR ISO 14040 - Gestão Ambiental - Avaliação do ciclo de vida - Princípios e estrutura (ABNT, 2009a) e pela NBR ISO 14044 - Gestão Ambiental - Avaliação do ciclo de vida - Requisitos e orientações (ABNT, 2009b), que substituíram e cancelaram as normas NBR ISO 14041, NBR ISO 14042 e NBR ISO 14043. A $\mathrm{ACV}$, em termos metodológicos, é composta das seguintes etapas: definição do objetivo e escopo; análise de inventário [ICV], avaliação de impacto ambiental [AICV] e interpretação (ABNT, 2009b).

O objetivo estabelece a aplicação pretendida, as razões para a execução do estudo, o público-alvo e se existe a intenção de utilizar os resultados em afirmações comparativas a serem publicadas. O escopo inclui a definição da abrangência do estudo, bem como parâmetros decisivos para as demais etapas, tais como sistema de produto (conjunto de unidades de processos, com fluxos elementares e de produto, que desempenha uma ou mais funções e que modela o ciclo de vida de um produto), unidade funcional (desempenho quantificado de um sistema para utilização como unidade de referência) e fronteira do sistema (conjunto de critérios que especificam quais processos elementares fazem parte do sistema de produto). (ABNT, 2009a). Uma vez que a ACV é uma técnica iterativa, o escopo do estudo pode necessitar de modificação enquanto o estudo estiver sendo conduzido, isto é quando informações adicionais forem coletadas nas etapas seguintes da $\mathrm{ACV}$.

A análise do inventário do ciclo de vida (ICV), segundo a norma NBR ISO 14040 (ABNT, 2009a), envolve a coleta de dados e procedimentos de cálculo para quantificar as entradas e saídas relevantes de um sistema de produto. Em um ICV todos os fluxos correspondentes a uma dada unidade funcional do sistema em estudo são contabilizados para o ciclo de vida do processo ou produto. Desse inventário resulta uma quantidade muito grande de dados numéricos que, para serem manipuláveis e interpretáveis têm que ser agrupados por meio de fatores de equivalência ou caracterização na fase seguinte.

$\mathrm{Na}$ terceira etapa da ACV, denominada Avaliação de Impacto do Ciclo de Vida (AICV), os dados coletados no inventário de ciclo de vida devem ser correlacionados a categorias de impacto, e para tanto é necessária a seleção e aplicação de um dos métodos de AICV internacionalmente reconhecidos e tecnicamente validados. Em linhas gerais, estes métodos dividem-se em ponto médio (midpoint) e em ponto final (endpoint); e diferenciam-se na forma de abordagem e na amplitude de suas categorias de impacto. Os métodos de ponto médio (midpoint) envolvem categorias de impacto, tais como aquecimento global, potencial de depleção da camada de ozônio, potencial de acidificação, potencial de eutrofização, ecotoxicidade e toxicidade humana, entre outros. Os métodos de ponto médio partem das substâncias para estimar seu potencial de impacto. Os métodos de ponto final (endpoint) baseiam-se em estudos epidemiológicos para determinar a nocividade das substâncias emitidas. São categorias mais amplas como saúde humana, recursos 
naturais e qualidade dos ecossistemas (Thrane e Schmidt, 2006).

Referente à abordagem da ACV, esta pode ser tanto atribucional quanto consequencial. A ACV atribucional descreve os impactos e fluxos de recursos em um sistema atribuído a certa quantidade de unidade funcional; por sua vez, a ACV consequencial estima como os impactos e fluxos de recursos de um sistema se alteram em decorrência de uma mudança na saída da unidade funcional (Thomassen et al, 2008).

A ACV pode ser aplicada tanto de forma prospectiva, como instrumento de apoio à tomada de decisões, quanto retrospectiva, com a finalidade de avaliar cargas ambientais de produtos e serviços já implantados. Entre suas aplicações, os autores Wenzel, Hauschild e Alting (1997), citam desde planos de ação comunitários desenvolvidos pelas autoridades, até escolhas em design pelo setor produtivo e possíveis rótulos ambientais para associações de consumidores.

\section{MÉTODO DE PESQUISA}

O levantamento do estado da arte referente o uso da ACV aplicada a tecnologias de remediação foi conduzido, com base no método de revisão bibliográfica sistemática. A revisão sistemática é um método de pesquisa específica, desenvolvida formalmente, para levantamento e avaliação de evidências pertencentes a um determinado foco de pesquisa (Brereton et al., 2007; Pigosso, 2008). O objetivo desta pesquisa foi o de identificar e analisar o uso do método de ACV aplicado a tecnologias de remediação de áreas contaminadas. Convém ressaltar que o levantamento de dados procedeu-se até o mês de dezembro de 2011.

A figura 1 apresenta uma síntese dos critérios adotados. Os critérios envolveram o estabelecimento de palavras-chave para a busca de informações; a definição dos meios e plataformas de busca (bases de dados); definição das informações a serem extraídas dos estudos levantados; determinação dos critérios a serem utilizados; classificação dos artigos de ACV em tecnologias de remediação, bem como sua sistematização.

Os resultados obtidos foram organizados e avaliados em relação ao número de publicações encontradas (artigos de periódicos e outras fontes), compilação da produção em razão dos periódicos de publicação, autores, país de origem e ano de publicação.

As informações levantadas referentes às estruturas de ACV foram organizadas de acordo as etapas da ACV descritas na norma NBR ISO 14040 (ABNT, 2009a). Desta forma, os estudos considerados relevantes de acordo com os critérios da pesquisa foram ordenados considerando o objetivo e escopo, inventário, avaliação de impacto do ciclo de vida e interpretação. Em relação ao objetivo e escopo, tentou-se identificar a finalidade do estudo, tipo de contaminantes e tecnologias e características da unidade funcional estabelecidas. Nos inventários dos estudos, foram identificados os principais parâmetros considerados e medidos. As principais categorias de impacto e modelos de avaliação mais adotados também foram identificadas.

\begin{tabular}{|l|l|}
\hline \multicolumn{1}{|c|}{ Informações e critérios } & \multicolumn{1}{c|}{ Descrição } \\
\hline Palavras-chave (inglês) & $\begin{array}{l}\text { LCA and remediation; life cycle assessment and remediation; } \\
\text { green remediation; sustainable remediation }\end{array}$ \\
\hline Palavras-chave (português) & $\begin{array}{l}\text { ACV e remediação, avaliação do ciclo de vida e remediação, } \\
\text { remediação verde e sustentável. }\end{array}$ \\
\hline Plataformas de busca & $\begin{array}{l}\text { Scopus, Web of science, Scielo, Ibict, Google e Science } \\
\text { Direct. }\end{array}$ \\
\hline Fontes de publicação & $\begin{array}{l}\text { Artigos de periódicos, artigos de congresso, livros, } \\
\text { dissertações e teses. }\end{array}$ \\
\hline Período e origem das publicações & Ano e país. \\
\hline Estrutura da ACV & $\begin{array}{l}\text { Definição do objetivo e escopo, parâmetros de inventário, } \\
\text { categorias de impacto para todos os contaminantes e técnicas } \\
\text { de remediação. }\end{array}$ \\
\hline
\end{tabular}

Figura 1 - Síntese das informações e critérios adotados

Fonte: Adaptado de Cunha, 2012

Revista de Gestão Social e Ambiental - RGSA, São Paulo, v. 6, n. 2, p. 3-18, maio/ago. 2012. 


\section{ANÁLISE E DISCUSSÃO DOS RESULTADOS}

Esta seção apresenta a síntese da produção de conhecimento sobre ACV aplicada a remediação e o detalhamento metodológico adotado por diferentes autores sobre a aplicação da ACV para tecnologias de remediação.

\subsection{Síntese da produção localizada referente a ACV aplicada a remediação}

Com base em revisão sistemática realizada, conforme os critérios apresentados na figura 1 , foram identificadas trinta e sete publicações nos temas envolvendo remediação e ACV, remediação e sustentabilidade e remediação e outros métodos de avaliação. Entre estas, trinta (Tabela 1) são artigos publicados em periódicos indexados e sete de outras fontes, tais como relatórios técnicos e tese de doutorado (Beinat e Nijkamp, 1997; Beinat e Van Drunen, 1997; Cunha, Ruiz e Teixeira, 2010a; 2010b; DNRA, 2000; Lemming, 2010; Lesage, Deschênes, Samson, 2010).

O banco de dados de teses e as dissertações do Instituto Brasileiro de Informação em Ciências e Tecnologia (IBICT) e a base de artigos e periódicos Scielo foram consultados e não foi encontrada nenhuma produção científica para os temas pesquisados no Brasil. As produções existentes em evento nacional e internacional do Brasil são oriundas de Cunha, Ruiz e Teixeira (2010a; 2010b), que foram considerados na contagem. Cunha (2012) refere-se a dissertação de mestrado, não considerada na contagem. Ressalta-se que os critérios de busca estabelecidos não permitiram levantar a produção científica em eventos na área, a não ser aquela disponibilizada via internet. Desta forma, esta pesquisa não conseguiu avaliar sistematicamente a produção científica do uso de ACV aplicado a tecnologias de remediação a partir de anais de eventos tanto nacional quanto internacional.

A Tabela 1 apresenta a distribuição da produção localizada por periódico, fator de impacto [FI]; números de artigos publicados em cada periódico e a citação da produção. A produção localizada em periódicos indexados apresentou-se distribuída em quinze periódicos diferentes. $\mathrm{O}$ periódico que publicou o maior número de artigos sobre ACV e remediação foi o International Journal of Life Cycle Assesment [IJLCA], no qual foram localizados seis, seguido do Journal of Hazardous Materials [JHM], com quatro e do Environmental Science \& Technology [EST] com três artigos.

Tabela 1 - Distribuição de artigos por periódicos, fator de impacto do periódico, quantidades e autores

\begin{tabular}{|l|c|c|l|}
\hline \multicolumn{1}{|c|}{ Nome do Periódico (Sigla) } & $\begin{array}{c}\text { FI } \\
\mathbf{2 0 1 0}\end{array}$ & $\begin{array}{c}\mathbf{N}^{\circ} \\
\text { Artigos }\end{array}$ & \multicolumn{1}{c|}{ Autores (ano) } \\
\hline $\begin{array}{l}\text { 1.The International Journal of Life } \\
\text { Cycle Assessment (IJLCA) }\end{array}$ & 3,148 & 6 & $\begin{array}{l}\text { Cadotte, Deschênes e Samson (2007); Lemming, } \\
\text { Hauschild, e Bjerg (2010); Lesage, Ekvall, Deschênes } \\
\text { e Samson (2007a); Lesage, Ekvall, Deschênes e } \\
\text { Samson (2007b);Toffoletto, Deschênes e Samson } \\
\text { (2005); Volkwein, Hurting e Klöpffer (1999). }\end{array}$ \\
\hline $\begin{array}{l}\text { 2. Journal of Hazardous Materials } \\
\text { (JHM) }\end{array}$ & 3,723 & 4 & $\begin{array}{l}\text { Bonano, Apostolakis, Salter, Ghassemi e Jennings } \\
\text { (2000); Hu, Zhu e Ding (2011); Inoue e Katayama } \\
\text { (2011); Morais e Delerue-Matos (2010). }\end{array}$ \\
\hline $\begin{array}{l}\text { 3. Science of the Total Environment } \\
\text { (STE) }\end{array}$ & 3,190 & 4 & $\begin{array}{l}\text { Onwubuy et al. (2009); Pollard et al. (2008); Pollard } \\
\text { et al. (2004); Sparrevik, Saloranta e Correlissen } \\
\text { (2011). }\end{array}$ \\
\hline $\begin{array}{l}\text { 4. Biomass \& Bioenergy (BB) } \\
\text { 5. Environmental Science \& }\end{array}$ & 3,840 & 2 & $\begin{array}{l}\text { Suer \& Andersson-Sköld (2011); Wittters et al. } \\
\text { (2012) }\end{array}$ \\
\hline $\begin{array}{l}\text { Technology (EST) } \\
\text { Civil Engineers Geotechnical } \\
\text { Engineering (PICEGE) }\end{array}$ & 4,827 & 3 & $\begin{array}{l}\text { Higgins e Olson, (2009); Lemming et al. (2010); Mak } \\
\text { e Lo (2011). }\end{array}$ \\
\hline
\end{tabular}


Avaliação do Ciclo de Vida (ACV) aplicada a remediação de áreas contaminadas

\begin{tabular}{|c|c|c|c|}
\hline $\begin{array}{l}\text { 7. Advances in Environmental } \\
\text { Research }^{1}(A E R)\end{array}$ & 2,596 & 1 & Blanc, Métivier-Pignon, Gourdan e Rousseaux (2004). \\
\hline $\begin{array}{l}\text { 8. Journal of Cleaner Production } \\
(J C P)\end{array}$ & 2,425 & 1 & Hermann, Kroeze e Jawjit (2007) \\
\hline $\begin{array}{l}\text { 9. Journal of Environmental } \\
\text { Management (JEM) }\end{array}$ & 2,596 & 1 & Kielenniva, Antikainen e Sorvari (2011). \\
\hline $\begin{array}{l}\text { 10. Journal of Contaminant Hydrology } \\
(\mathrm{JCH})\end{array}$ & 2,124 & 1 & Bayer e Finkel (2006). \\
\hline 11. Remediation Journal $(R J)$ & & 1 & Baker, Smith e Woodward (2009). \\
\hline $\begin{array}{l}\text { 12. Environmental Toxicology and } \\
\text { Chemistry (ETC) }\end{array}$ & 3,025 & 1 & $\begin{array}{l}\text { Diamond, Page, Campbell, McKenna e Lall (1999); } \\
\text { Page, Diamond, Campbell e Mckenna (1999). }\end{array}$ \\
\hline $\begin{array}{l}\text { 13. Human and Ecological Risk } \\
\text { Assessment }(\text { HERA) }\end{array}$ & 1,486 & 1 & Godin, Ménard, Hains, Deschênes e Samson (2004). \\
\hline $\begin{array}{l}\text { 14. Soil and Sediment Contamination: } \\
\text { An International Journal (SSC) }\end{array}$ & 0,808 & 1 & Suer, Nilsson-Paledal e Norrman (2004). \\
\hline $\begin{array}{l}\text { 15. Chemical Engineering Progress }{ }^{2} \\
(C E P)\end{array}$ & & 1 & Vignes (2001). \\
\hline Total de Artigos & & 30 & \\
\hline
\end{tabular}

Periódico incorporado em 2012 ao Journal of Environmental Management

${ }^{2}$ Revista técnica

${ }^{3}$ Disponível online em 2011 (in pres)

Fonte: Dados da pesquisa

Os periódicos que publicaram artigos envolvendo ACV e tecnologias de remediação possuem, em sua grande maioria, fator de impacto acima de 1,0, o que representa uma repercussão científica internacional expressiva. Desta forma, esse tema foi considerado relevante para os meios de divulgação científica de impacto.

Durante os anos 2010 e 2011 ocorreu o maior número de publicações, quinze das trinta publicações, o que pode indicar uma tendência ao aumento do número de iniciativas, a considerar as questões ambientais na escolha de tecnologias de remediação. O Canadá foi o país que publicou a maior quantidade de artigos, com seis artigos; seguido da China, Suécia e Alemanha, com dois artigos publicados em cada país.

\subsection{ACV aplicada a tecnologias de remediação}

Entre os estudos de ACV de tecnologias de remediação levantados, treze foram considerados com a aplicação do método completo (Figura 1), isto é, contemplando as quatro etapas da ACV descritas nas normas ABNT NBR ISO 14040 e 14044. Blanc et al.(2004) aplicaram os dados do inventário do ciclo de vida em análise multicritério. A Figura 2 apresenta um sistema de produto genérico para ACV aplicada a tecnologias de remediação, proposto por Diamond et al. (1999).

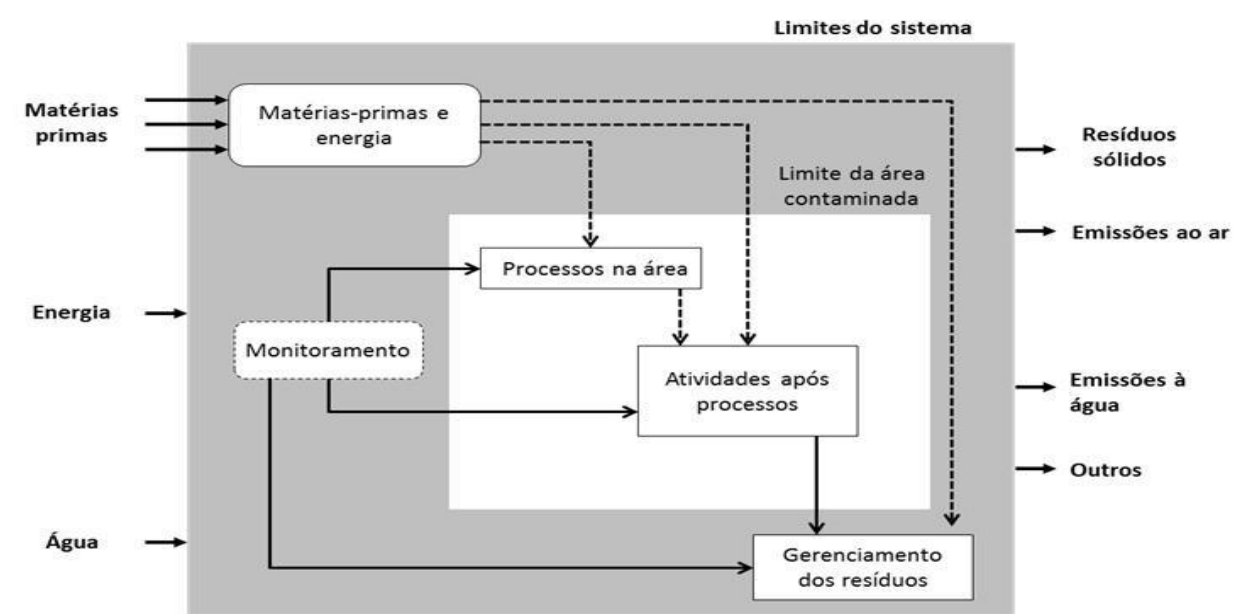

Figura 2 - Diagrama de fluxos representando a relação entre estágios do ciclo de vida e itens do inventário Fonte: Adaptado de Diamond et al. (1999) 
A ACV de tecnologias de remediação contempla os estágios e subestágios do processo e as entradas e saídas. As entradas consideradas são matérias-primas, água e energia e as saídas emissões ao ar, solo e água, resíduos, liberação de calor e solo tratado. Para Bayer e Finkel (2006), em um estudo comparativo, a qualidade do design técnico e dos parâmetros de desempenho dependem fundamentalmente da experiência na tecnologia e conhecimento da área contaminada. Se uma tecnologia for implementada, as características de seu desempenho são conhecidas mais precisamente que uma alternativa hipotética. Além disso, para uma medição realizada, o consumo associado de material e energia é aparente, enquanto alternativas potenciais podem ser mensuradas apenas teoricamente. Os estudos de caso conduzidos mostram que o uso da ACV em processos de remediação é efetivo em situações específicas, que diferentes possibilidades de remediação são disponíveis financeiramente, legalmente e tecnicamente (Volkwein, Hurting \& Klöpffer, 1999).

Os estudos sobre ACV de tecnologias de remediação localizados foram, na sua maioria, estudos prospectivos, conforme mostra a figura 3. Ou seja, os estudos foram realizados antes da implantação das tecnologias de remediação, com o objetivo de considerar a variável ambiental na seleção entre tecnologias (Blanc et al., 2004; Cadotte, Deschênes e Samson, 2007; Godin et al., 2004; Higgins e Olson, 2009; Lemming et al., 2010; Tofoletto, Deschênes e Samson, 2005; Volkwein, Hurting e Klöpffer, 1999).

\begin{tabular}{|c|c|c|}
\hline Título do Artigo & País & Autores (ano) \\
\hline $\begin{array}{l}\text { 1. Biofuel or excavation? - life cycle assessment (LCA) of soil } \\
\text { remediation options. }\end{array}$ & Suécia & $\begin{array}{l}\text { Suer e Andersson-Sköld } \\
(2011)\end{array}$ \\
\hline $\begin{array}{l}\text { 2. Environmental life-cycle comparisons of two polychlorinated } \\
\text { biphenylremediation technologies: Incineration and base catalyzed } \\
\text { decomposition. }\end{array}$ & China & Hu, Zhu e Ding (2011) \\
\hline $\begin{array}{l}\text { 3. Environmental life cycle assessment of permeable reactive barriers: } \\
\text { Effects of Construction Methods, Reactive Materials and Groundwater } \\
\text { Constituents }\end{array}$ & China & Mak e Lo (2011) \\
\hline $\begin{array}{l}\text { 4. Environmental impacts of remediation of a trichloroethene- } \\
\text { contaminated site: life cycle assessment of remediation alternatives. }\end{array}$ & $\begin{array}{l}\text { Dinamarca, } \\
\text { Canada }\end{array}$ & Lemming et al. (2010) \\
\hline $\begin{array}{l}\text { 5. Life-cycle case study comparison of permeable reactive barrier } \\
\text { versus pump-and-treat remediation. }\end{array}$ & EUA & Higgins e Olson, (2009) \\
\hline $\begin{array}{l}\text { 6. Selection of a remediation scenario for a diesel-contaminated site } \\
\text { using LCA. }\end{array}$ & Canada & $\begin{array}{l}\text { Cadotte, Deschênes e } \\
\text { Samson (2007) }\end{array}$ \\
\hline $\begin{array}{l}\text { 7. Environmental Assessment of Brownfield Rehabilitation Using Two } \\
\text { Different Life Cycle Inventory Models: Part 2: Case Study. }\end{array}$ & Canada & $\begin{array}{l}\text { Lesage, Ekvall, } \\
\text { Deschênes, e Samson } \\
(2007 \mathrm{~b})\end{array}$ \\
\hline $\begin{array}{l}\text { 8. Life cycle assessment of active and passive groundwater } \\
\text { remediation technologies. }\end{array}$ & Alemanha & Bayer e Finkel (2006) \\
\hline 9. LCA of ex-situ bioremediation of diesel-contaminated soil. & Canada & $\begin{array}{l}\text { Toffoletto, Deschênes e } \\
\text { Samson (2005) }\end{array}$ \\
\hline $\begin{array}{l}\text { 10. Combined Use of Life Cycle Assessment and Groundwater } \\
\text { Transport Modeling to Support Contaminated Site Management. }\end{array}$ & Canada & Godin et al. (2004) \\
\hline $\begin{array}{l}\text { 11. Life cycle assessment as a tool for controlling the development of } \\
\text { technical activities: application to the remediation of a site contaminated } \\
\text { by sulfur. }\end{array}$ & França & Blanc et al. (2004) \\
\hline $\begin{array}{l}\text { 12. Life-cycle framework for assessment of site remediation options: } \\
\text { case study. }\end{array}$ & Canada & Page et al. (1999) \\
\hline 13. Life cycle assessment of contaminated sites re & Alemanha & $\begin{array}{l}\text { Volkwein, Hurting e } \\
\text { Klöpffer (1999) }\end{array}$ \\
\hline
\end{tabular}

Figura 3 - Estudos de caso - ACV aplicada a remediação

Fonte: Dados da pesquisa

A ACV prospectiva baseou-se no projeto das tecnologias consideradas para serem implantadas em uma determinada área contaminada (Figura 3), portanto contemplando estimativas de entradas e saídas, obtidas por cálculo estequiométrico ou mensuradas em piloto da aplicação das tecnologias na área. No caso de ACV retrospectiva, os cálculos foram feitos com base nas entradas 
e saídas documentadas na implantação e manutenção das tecnologias. Referente à abordagem da ACV, apenas o estudo realizado por Lesage et al. (2007b) realizou ACV consequencial, sendo o objetivo do estudo avaliar as consequências do uso posterior à remediação do local, compreendendo reabilitação para uso residencial, ou minimização da exposição. Os demais apresentaram abordagem atribucional, sendo, de forma geral, o objetivo dos estudos avaliar os impactos associados à contaminação e/ou a aplicação de certa(s) tecnologia(s) de remediação.

As áreas contaminadas que foram objeto de estudo em ACV foram impactadas, sobretudo, por hidrocarboneto policíclico aromático [HPA], avaliado em três estudos, e óleo mineral, diesel e cromo, avaliados em dois estudos. O contaminante HPA é classificado na ABNT NBR 10004 (2004) como resíduo classe I, isto é, resíduo perigoso. A emissão de HPA está associada a ações antrópicas, tais como derramamento de petróleo, queima de combustíveis e resíduos industriais (Sisinno, Pereira Netto, Rego e Lima, 2003).

\begin{tabular}{|c|c|c|c|}
\hline Tipo de ACV & Contaminante & Tecnologias & Autores \\
\hline $\mathrm{R}$ & $\begin{array}{l}\text { Óleo mineral; benzeno, tolueno, } \\
\text { etilbenzeno e xileno (BTEX) }\end{array}$ & $\begin{array}{l}\text { Fitorremediação; Escavação e } \\
\text { disposição }\end{array}$ & $\begin{array}{l}\text { Suer e Andersson-Skold } \\
(2011)\end{array}$ \\
\hline $\mathrm{R}$ & Bifenilas policloradas (PCBs) & $\begin{array}{l}\text { Incineração, Decomposição } \\
\text { catalisada por base }\end{array}$ & Hu, Zhu e Dingb (2011) \\
\hline $\mathrm{P}$ & Cromo VI e arsênio & Barreira reativa permeável & Mak e Lo, (2011) \\
\hline $\mathrm{P}$ & Tricloroeteno (TCE) & $\begin{array}{l}\text { Biorremediação; dessorção térmica; } \\
\text { Escavação, tratamento e disposição }\end{array}$ & Lemming et al. (2010) \\
\hline $\mathrm{P}$ & Compostos orgânicos voláteis & $\begin{array}{l}\text { Barreira permeável reativa; } \\
\text { bombeamento e tratamento }\end{array}$ & Higgins e Olson (2009) \\
\hline $\mathrm{P}$ & Diesel & $\begin{array}{l}\text { Bombeamento e tratamento; } \\
\text { atenuação natural; bioventilação; } \\
\text { oxidação química; remoção de óleo }\end{array}$ & $\begin{array}{l}\text { Cadotte, Deschênes e } \\
\text { Samson, (2007) }\end{array}$ \\
\hline $\mathrm{C}$ & $\begin{array}{l}\text { Hidrocarbonetos policíclicos } \\
\text { aromáticos (HPA); metais }\end{array}$ & Escavação e disposição & Lesage et al. (2007b) \\
\hline $\mathrm{R}$ & HPA, betume & $\begin{array}{l}\text { Bombeamento e tratamento; } \\
\text { Barreira permeável; Barreira } \\
\text { impermeável. }\end{array}$ & Bayer e Finkel (2006) \\
\hline $\mathrm{P}$ & $\begin{array}{l}\text { Aterro de borra residual de } \\
\text { produção de alumínio (SPL) }\end{array}$ & $\begin{array}{l}\text { Escavação e disposição on-site; } \\
\text { Escavação e tratamento da fração } \\
\text { SPL; Atenuação natural. }\end{array}$ & Godin et al. (2004) \\
\hline $\mathrm{P}$ & Diesel & Escavação e biopilha on-site & $\begin{array}{l}\text { Toffoletto, Deschênes e } \\
\text { Samson (2005) }\end{array}$ \\
\hline $\mathrm{P}$ & Enxofre & $\begin{array}{l}\text { Escavação e biolixiviação; } \\
\text { Escavação e disposição em aterro; } \\
\text { Escavação e contenção on-site; } \\
\text { Escavação e calagem. }\end{array}$ & Blanc et al. (2004) \\
\hline $\mathrm{R}$ & Chumbo & Escavação e disposição. & Page et al. (1999) \\
\hline $\mathrm{P}$ & HPA, óleo mineral e cromo & $\begin{array}{l}\text { Escavação e contenção on-site; } \\
\text { Escavação e remediação }\end{array}$ & $\begin{array}{l}\text { Volkwein, Hurting e } \\
\text { Klöpffer (1999) }\end{array}$ \\
\hline
\end{tabular}

$\mathrm{P}$ (prospectivo), R (retrospectiva) e C (consequencial)

Figura 4: Tipos de ACV, contaminantes, tecnologias e autores

Fonte: Dados da pesquisa

As tecnologias de remediação avaliadas diferiram entre os estudos, sendo as tecnologias mais avaliadas: escavação e disposição, bombeamento e tratamento, e barreira reativa permeável. Esses conjuntos de tecnologias foram citados cada um em três estudos, seguidos de escavação e contenção on-site e atenuação natural, avaliados em dois estudos. Entre os estudos analisados, sete realizaram ACV para alternativas tecnológicas ex situ, cinco para alternativas ex situ e in situ e apenas um estudou e contemplou apenas alternativa in situ. 
De forma geral, conforme apresentado por Toffoletto, Deschênes e Samson (2005), a unidade funcional dos estudos de ACV aplicados à tecnologia de remediação baseia-se em um montante de solo e/ou aquífero contaminado, uma concentração alvo de contaminação a ser atingida, e um determinado tempo de remediação. Considerando que o tempo de cada processo de remediação pode apresentar variações em razão da tecnologia e das características da contaminação, este parâmetro apresentou notável variação. Foram encontrados tanto estudos que consideraram dois anos (Cadotte, Deschênes e Samson, 2007; Toffoletto, Deschênes e Samson, 2005), como estudo que considerou 50 anos (Godin et al., 2004). Considerando que os equipamentos utilizados para remediação podem ser utilizados em outros sistemas de produto, essa foi a principal justificativa para exclusão de equipamentos e infraestrutura da fronteira dos estudos (Morais e Delerue-Matos, 2010).

A fonte e qualidade dos dados primários e/ou secundários dos estudos variaram conforme disponibilidade de dados. Blanc et al. (2004) utilizaram, para seu estudo, dados de remediação dos relatórios de consultores, e Lemming et al. (2010) coletaram dados em empresas. Bases de dados disponibilizadas nos softwares de ACV eventualmente também foram utilizadas nos estudos, principalmente para entradas, tais como energia e transporte.

Conforme apresentado por Lemming, Hauschild e Bjerg (2010), para alguns aspectos ambientais associados às tecnologias de remediação (materiais e serviços específicos), ainda são inexistentes inventários para ACV. Os autores citam, por exemplo, a produção de carvão ativado, utilizado em tratamento de vapor ou água em vários projetos de remediação, assim como produtos específicos de algumas tecnologias a serem adicionados ao solo, tais como culturas de microrganismos, ferro zero-valente, substratos, oxidantes químicos e outros, bem como serviços de laboratórios para análise de solos e aquífero.

Para a avaliação do impacto do ciclo de vida, a maior parte dos estudos utilizou metodologias de impacto de ponto médio (midpoint), sendo os métodos Environmental Development of Products (EDIP) e Tool for the Reduction and Assessment of Chemical and Other Environmental Impacts (TRACI) os mais incidentes, ambos utilizados em três estudos. O software mais utilizado foi o Simapro, em sete estudos.

A ACV aplicada a remediação de áreas contaminadas apresenta como particularidade a subdivisão dos impactos associados ao sistema de produto em: primários, secundários e terciários. Os impactos relacionados à contaminação da área são considerados primários, enquanto aqueles associados aos processos de remediação são definidos como secundários (Diamond et al., 1999; Godin et al., 2004; Lemming et al., 2010; Lesage et al., 2007b; Morais e Delerue-Matos, 2010; Page et al., 1999; Tofoletto, Deschênes e Samson, 2005; Volkwein, Hurting e Klöpffer, 1999). Conforme apresentado anteriormente por Lesage et al. (2007b) foi o único estudo a utilizar a abordagem da ACV consequencial, que contempla também os impactos da reabilitação da área, que, por sua vez, são definidos pelos autores como impactos terciários. Entre os estudos levantados na revisão, sete consideraram os impactos primários em seus estudos de $\mathrm{ACV}$, e todos consideraram os impactos secundários.

A categoria de impacto de maior incidência nos estudos de ACV em remediação foi o potencial de aquecimento global, analisada em onze dos treze estudos, seguida pelas categorias: potencial de depleção da camada de ozônio, potencial de acidificação e potencial de eutrofização, com incidência em nove estudos.

A interpretação da ACV deve identificar e discutir os elementos mais relevantes das tecnologias de remediação, bem como pode derivar sugestões que possibilitem o aprimoramento tecnológico das rotas de remediação. Conforme discutido por Bayer e Finkel (2006), a interpretação deve acompanhar todo o estudo da ACV, uma vez que a metodologia é iterativa. O uso da ACV em processos de remediação é efetivo em situações em que diferentes possibilidades de remediação são disponíveis financeiramente, legalmente e tecnicamente (Volkwein, Hurting e Klöpffer, 1999). A alta incidência nos estudos analisados da alternativa escavação decorre da maior facilidade em 
escavar e tratar ou dispor o material contaminado, frente a um tratamento in loco (in situ), sendo que, normalmente, a fonte de contaminação precisa ser isolada ou removida.

\section{CONSIDERAÇÕES FINAIS}

A ACV é um método que vem sendo utilizado para avaliar tecnologias de remediação. Na revisão sistemática realizada neste estudo, foram identificados 37 estudos, sendo 30 artigos publicados em periódicos internacionais de relevância na área. Entre os estudos localizados, 13 estudos aplicaram a ACV de forma completa, sendo sete para alternativas tecnológicas ex situ, cinco para alternativas ex situ e in situ e apenas um estudou e contemplou apenas alternativa in situ.

A aplicação da ACV nos estudos de caso pesquisados propiciou resultados para a tomada de decisão quanto à avaliação de impactos ambientais de tecnologias de remediação, segundo os autores pesquisados. Contudo, limitações acerca da complexidade do processo e carência de dados se destacam entre as fraquezas deste tipo de estudo. É preciso um alto grau de desenvolvimento e conhecimento das tecnologias envolvidas, bem como inventários específicos aos processos de remediação. Escolhas e inferências devem ser feitas ao longo da avaliação, o que o torna um método com alta dependência do nível de conhecimento da equipe responsável pela avaliação e/ou dos experts consultados.

\section{AGRADECIMENTOS}

Os autores agradecem o apoio recebido do BNDES pelo projeto "Desenvolvimento e Validação de Tecnologias para Remediação de Solo e Água Subterrânea Contaminados com Organoclorados" sob coordenação do IPT-SP e o apoio recebido do Fundo de Apoio a Pesquisa - FAP/UNINOVE. Os autores agradecem também o colega Mario Roberto dos Santos pela revisão de citações e referências do artigo.

\section{REFERÊNCIAS}

Associação Brasileira de Normas Técnicas [ABNT]. (2009a). NBR ISO 14040: Gestão ambiental Avaliação do ciclo de vida - Princípios e estrutura. Rio de Janeiro.

Associação Brasileira de Normas Técnicas [ABNT]. (2009b). NBR ISO 14044: Gestão ambiental Avaliação do ciclo de vida - Requisitos e orientações. Rio de Janeiro.

Baker, C. B., Smith, L. M., Woodward, D. S. (2009). The sustainable remediation forum. Remediation Journal, 19 (4), 135-139.

Bayer, P. , Finkel, M. (2006). Life cycle assessment of active and passive groundwater remediation technologies. Journal of Contaminant Hydrology, 83(3/4), 171-199.

Beinat, E., Nijkamp, P. (1997). Environmental rehabilitation: efficiency and effectiveness in soil remediation. Amsterdam: Faculteit der Economische Wetenschappen en Econometric.

Beinat, E., Van Drunen, M. A. (Ed.). (1997). The REC decision support system for comparing soil remediation options: a methodology based on risk reduction, environmental merit and costs. University Amsterdam / Institute for Environment Studies.

Blanc, A., Métivier-Pignon, H., Gourdan, R., Rousseaux, P. (2004). Life cycle assessment as a tool for controlling the development of technical activities: application to the remediation of a site 
contaminated by sulfur. Advances in Environmental Research. 8 (3-4), 613-627.

Bonano, E. J., Apostolakis, G. E., Salter P. F., Ghassemi, A. , Jennings, S. (2000). Application of risk assessment and decision analysis to the evaluation, ranking and selection of environmental remediation alternatives. Journal of Hazardous Materials. 71 (1-3), 35-57.

Brereton, P., Kitchenham, B. A., Budgen, D., Turner, M., Khalil, M. (2007). Lessons from applying the systematic literature review process within the software engineering domain. The Journal of Systems and Software. 80 (4), 571-583.

Cadotte, M., Deschênes, L. \& Samson, R. (2007). Selection of a remediation scenario for a dieselcontaminated site using LCA. The International Journal of Life Cycle Assessment. 12 (4), 239-251.

Companhia Ambiental do Estado de São Paulo - CETESB (2007). Decisão de Diretoria $n^{o}$ $103 / 2007 / C / E$, de 22 de junho de 2007. Dispõe sobre o procedimento para gerenciamento de áreas contaminadas. Recuperado em 30 novembro, 2011, de: http://www.cetesb.sp.gov.br/Solo/areas_contaminadas/proced_gerenciamento_ac.pdf

Companhia Ambiental do Estado de São Paulo - CETESB. (2011). Texto explicativo, relação de áreas contaminadas e reabilitadas no Estado de São Paulo. Recuperado em 23 abril, 2012, de: http://www.cetesb.sp.gov.br/userfiles/file/areas-contaminadas/2011/texto-explicativo.pdf

Conselho Nacional de Meio Ambiente [CONAMA]. Resolução $n^{\circ} 420$, de 28 de dezembro de 2009. Dispõe sobre critérios e valores orientadores de qualidade do solo quanto à presença de substâncias químicas e estabelece diretrizes para o gerenciamento ambiental de áreas contaminadas por essas substâncias em decorrência de atividades antrópicas. Recuperado em 20 junho, 2011, de: http://www.mma.gov.br/port/conama/legiabre.cfm?codlegi $=620$

Côrtes, P. L, Alves Filho, A. P., Ruiz, M. S. \& Teixeira, C. E. (2011). A deposição de resíduos industriais organoclorados no litoral do Estado de São Paulo: um estudo de caso. RAI : Revista de Administração e Inovação. 8 (2), 132-163.

Cunha, A. C. L. L. (2012). Avaliação do Ciclo de Vida (ACV) de tecnologias de remediação: contextualização e proposta de aplicação para uma área contaminada por Hexaclorociclohexano $(\mathrm{HCH})$. Dissertação de mestrado. Instituto de Pesquisas Tecnológicas do Estado de São Paulo IPT, São Paulo, Brasil.

Cunha, A. C. L. L., Ruiz, M. S. \& Teixeira, C. E. (2010a). Environmental assessment of remediation technologies: an analytical framework for a hexachlorocyclohexane (hch) contaminated site in Brazil. Proceedings of the International Conference Hazardous and Industrial Waste Management, Chania, Crete, Greece, 2, 1-8.

Cunha, A. C. L. L., Ruiz, M. S. \& Teixeira, C. E. (2010b). Sustentabilidade na seleção de tecnologias de remediação de áreas contaminadas. Anais do Congresso Brasileiro em Gestão do Ciclo de Vida de Produtos e Serviços, Florianópolis, UFSC, 2,1-6.

Danish National Railway Agency - DNRA (2000). Danish State Railways. Environmental/economic evaluation and optimising of contaminated sites remediation method to involve environmental assessment. Copenhagen.

Diamond, M. L., Page, C. A., Campbell, M., McKenna, S. \& Lall, R. (1999). Life-cycle framework 
for assessment of site remediation options: method and generic survey. Environmental Toxicology and Chemistry. 18 (4), 788-800.

Galdino, C. A. B., Santos, E. M., Pinheiro, J. I. Marques Junior, S., Ramos, R. E. B. (2004). Passivo ambiental: revisão teórica de custos na indústria do petróleo. Produção.14 (1), 54-63.

Godin, J., Ménard, J. F., Hains, S., Deschênes, L. \& Samson, R. (2004). Combined use of life cycle assessment and groundwater transport modeling to support contaminated site management. Human and Ecological Risk Assessment. 10 (6), 1099-1116.

Harbottle, M. J., Al-Tabbaa, A. \& Evans, C. W. (2008a). Sustainability of land remediation. Part I: overall analysis. Proceedings of the Institution of Civil Engineers Geotechnical Engineering, London,161 (2), 75-92.

Harbottle, M. J., Al-Tabbaa, A. \& Evans, C. W. (2008b). Sustainability of land remediation. Part II: impact assessment. Proceedings of the Institution of Civil Engineers Geotechnical Engineering, London, 161 (3), 117-127.

Hermann, B., Kroeze, C. \& Jawjit, W. (2007). Assessing environmental performance by combining life cycle assessment, multi-criteria analysis and environmental performance indicators. Journal of Cleaner Production.15 (18), 1787-1796.

Higgins, M. R. \& Olson, T. M. (2009). Life-cycle case study comparison of permeable reactive barrier versus pump-and-treat remediation. Environmental Science \& Technology.43 (24), 94329438.

Hu, X., Zhu, J. \& Ding Q. (2011). Environmental life-cycle comparisons of two polychlorinated biphenyl remediation technologies: incineration and base catalyzed decomposition. Journal of Hazardous Materials, 191 (1-3), 258-268.

Inoue, Y. \& Katayama, A. (2011). Two-scale evaluation of remediation technologies for a contaminated site by applying economic input-output life cycle assessment: Risk-cost, risk-energy consumption and risk-CO ${ }_{2}$ emission. Journal of Hazardous Materials. 192 (3), 1234-1242.

Kielenniva, N., Antikainen, R. \& Sorvari, J. (2011). Measuring eco-efficiency of contamined soil management at the regional level. Journal of Environmental Management.1-10.

Lemming, G. (2010). Environmental assessment of contaminated site remediation in a life cycle perspective. (Doctoral dissertation, 2010). Department of Environmental Engineering, Technical University Of Denmark, Lyngby.

Lemming, G., Hauschild, M. Z. \& Bjerg P. L. (2010). Life cycle assessment of soil and groundwater remediation technologies: literature review. The International Journal of Life Cycle Assessment. 15 (1), 115-127.

Lemming, G., Hauschild, M. Z., Chambon, J., Binning, P. J., Bulle, C., Margni, M., et al., (2010). Environmental impacts of remediation of a trichloroethene-contaminated site: life cycle assessment of remediation alternatives. Environmental Science \& Technology. 44 (23), 9163-9169.

Lesage, P. Deschênes, L., Samson, R. (2010). Environmental assessment of 
brownfieldrehabilitation using life cycle assessment - how site reuse trumps it all, Green Remediation, Amherst (USA), June 16

Lesage, P., Ekvall, T., Deschênes, L. \& Samson. (2007a). Environmental assessment of brownfield rehabilitation using two different life cycle inventory models: Part 1: methodological approach. The International Journal of Life Cycle Assessment. 12 (6), 391-398.

Lesage, P., Ekvall, T., Deschênes, L. \& Samson. (2007b). Environmental assessment of brownfield rehabilitation using two different life cycle inventory models: Part 2: case study. The International Journal of Life Cycle Assessment. 12 (7), 497-512.

Mak, M. S. H. \& Lo, I. M. C. (2011) Environmental life cycle assessment of permeable reactive barriers: effects of construction methods, reactive materials and groundwater constituents. Environmental Science Technology, 45 (23), 10148-10154.

Morais, S. A., Delerue-Matos, C. (2010). A perspective on LCA application in site remediation services: Critical review of challenges. Journal of Hazardous Materials, 175 (1-3), 12-22.

Oliveira, R. M., Bastos, L. H. P., Dias, A. E. X. O., Silva, S. A., \& Moreira, J. C. (2003). Concentração residual de hexaclorociclohexano em área contaminada na Cidade dos Meninos, Duque de Caxias, Rio de Janeiro, Brasil, após tratamento com óxido de cálcio. Cadernos de Saúde Pública. 19 (2), 447-453.

Onwubuya, K., Cundy, A., Puschenreiter, M., Kumpiene, J., Bone, B., Greaves, J., et al., (2009). Developing decision support tools for the selection of "gentle" remediation approaches. Science of the Total Environment. 407 (24), 6132-6142.

Page, C. A., Diamond, M. L., Campbell, M. \& Mckenna, S. (1999). Life-cycle framework for assessment of site remediation options: case study. Environmental Toxicology and Chemistry.18 (4), 801-810.

Phillips, A. S., Hung, Y. T., \& Bosela, P. A. (2007). Love Canal tragedy. Journal of Performance of Constructed Facilities. 21(4), 131-319.

Pigosso, D. C. A. (2008). Integração de métodos e ferramentas do ecodesign ao processo de desenvolvimento de produtos. Monografia (Conclusão de Curso em Engenharia Ambiental) Escola de Engenharia de São Carlos, Universidade de São Paulo.

Pollard, S. J. T., Brookes, A., Earl, N., Lowe, J., Kerrney, T. \& Nathanail, C. P. (2004). Integrating decision tools for the sustainable management of land contamination. Science of the Total Environment. 325 (1-3), 15-28.

Pollard, S. J. T., Davies, G. J., Coley, F. \& Lemon, M. (2008). Better environmental decision making — recent progress and future trends. Science of the Total Environment. 400 (1-3), 20-31.

São Paulo. Lei $n^{\circ} 13.577$, de 8 de julho de 2009. Dispõe sobre diretrizes e procedimentos para a proteção da qualidade do solo e gerenciamento de áreas contaminadas. Recuperada em 21 junho, 2011, de:

http://www.al.sp.gov.br/repositorio/legislacao/lei/2009/lei\%20n.13.577,\%20de\%2008.07.2009.htm

Sharma, H. D. \& Reddy, K. R. (2004). Geoenvironmental engineering: site remediation, waste 
containment, and emerging waste management technologies. Hoboken, New Jersey: John Wiley \& Sons.

Sisinno, C. L. S., Pereira Netto, A. D., Rego, E. C. P \& Lima, G. S. V. (2003). Hidrocarbonetos policíclicos aromáticos em resíduos sólidos industriais: uma avaliação preliminar do risco potencial de contaminação ambiental e humana em áreas de disposição de resíduos. Cad. Saúde Pública [online], 19 (2), 671-676.

Sparrevik, M., Saloranta, T., Cornelissen, G., Eek, E., Fet, A. M., Breedveld, G. D., et al., (2011). Use of Life Cycle assessments to evaluate the environmental footprint of contaminated sediment remediation. Environmental Science \& Tecnology. 45, 4235-4241.

Suer, P., Andersson-Sköld, Y. (2011). Biofuel or excavation? - Life cycle assessment (LCA) of soil remediation options. Biomass and Bioenergy. 35 (2), 969-981.

Suer, P., Nilsson-Paledal, S., Norrman, J. (2004). LCA for site remediation: a literature review. Soil \& Sediment Contamination: an International Journal. 13 (4), 415-425.

Thomassen, M.A, Dalgaard, R., Heijungs, R., Boer, I. (2008) Attributional and consequential LCA of milk production. International Journal of Life Cycle Assesment. 13, 339-349.

Thrane, M., Schmidt, J. (2006) Life cycle assessment. In: Tools for sustainable development. Aalborg: Department of Development and Planning, Aalborg University.

Toffoletto, L., Deschênes, L., Samson, R. (2005). LCA of ex-situ bioremediation of dieselcontaminated soil. The International Journal of Life Cycle Assessment.10 (6), 406-416.

Vignes, R. P. (2001). Use limited life-cycle analysis for environmental decision-making. Chemical Engineering Progress. 97 (2), 40-54.

Volkwein, S., Hurting, H. W. \& Klöpffer, W. (1999). Life cycle assessment of contaminated sites remediation. The International Journal of Life Cycle Assessment. 4 (5), 263-274.

Wenzel, H., Hauschild, M. \& Alting, L. (1997). Environmental assessment of products: volume 1: methodology, tools and case studies in product development. London: Chapman \& Hall.

Wittters, N., Mendelsohn, R. O., Van Slycken, S., Weyens, N., Schreurs, E., Meers, E., et al,. (2012). Phytoremediation, a sustainable remediation technology? Conclusions from a case study. I: Energy production and carbon dioxide abatement. Biomass \& Bioenergy, 39, 454-469.

Data do recebimento do artigo: 24/03/2012

Data do aceite de publicação: 23/08/2012 